\title{
IL PENDOLO ORIZZONTALE COME CLINOMETRO
}

\section{Pietro Caloi}

1. - Nella requistrazione ollica, si sa che per piecoli angoli di rotazione W, si ha

$$
\boldsymbol{d}=\frac{\lrcorner}{20},
$$

dove $\lrcorner$ i l'anpiezza registrata $6 D$ la distanzal specchio-registratore. Se si può valutare con sicurezza $\frac{1}{10}$ di $\mathrm{mm}$ di $\lrcorner$, ciò vuol dire che per $D=1000 \mathrm{~mm}$, il limite di certezza dell'angolo W ̀̀

$$
\text { (1) }=\text { ca. } 10^{\prime \prime}
$$

Nella teoria elementare dei pencioli orizzontali, se $i$ is l'inclinazione dell'asee di rotazione dello strumento sulla verticale, $T$ il periodo dello strumento ed $l_{\mathrm{r}}$ la lungheza del pendolo ridotto, si ha

$$
T=2 \pi \sqrt{\frac{l_{\mathrm{r}}}{g \sin i}},
$$

c. per piccoli valori di $i$.

$$
T=2 \pi / \frac{\overline{l_{\mathrm{r}}}}{{\overline{\mathrm{B}^{i}}}^{i}} .
$$

Se $l_{\mathrm{r}}=\mathrm{cm} 12, T=25$ icomune pendolo Galitzin si hat

$$
\begin{aligned}
i & =\frac{1}{300} \text { circa. in valore assoluto } \\
& =159^{\prime \prime} \text { in arco. }
\end{aligned}
$$

Sia $O P$ l'asse del pendolo (fir. 1 . Se si provoca una rotazione intorno arl un asse orizzontale per $O$ di un piccolo angolo ul', una sfera con centro $O$ sarà intercetlata in $Z$ dalla verticale, in $P$ dall'asse del pendolo $e$ in $P^{\prime}$ dal nuovo asse.

Dal piccolo triangolo sferico $Z P P^{\prime}$ si hia

$$
\operatorname{tang} y^{\prime}=\sin i \operatorname{tang} \Phi ;
$$


c. per piccoli angroli.

$$
\text { 小 }:=\frac{\psi}{i} \text {. }
$$

Dalla [2] consegue allora

$$
\text { 小 }=1300 \% \text {. }
$$

Per il limite di cerlezza di $10^{\prime \prime}$ cirea, nella determinazione del.

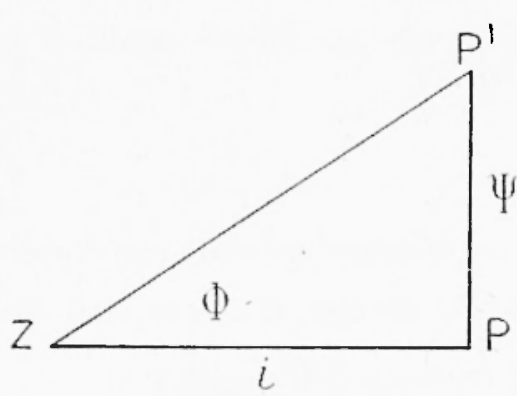

Fig. 1 l'anguolo di spostamento di un pendolo orizzontale dalla sua posiziothe di equilibrio, usamelo registrazione olliea dirella. il limile per la delerminazione delle ontulazioni fangolo d’inclinazione del stolof y' - variazome della direzione della verticale apparente .... diviene. per $T=25^{\circ}$ ed $l_{\mathrm{r}}=120 \mathrm{~mm}$.

$$
10^{\prime \prime} 1300=00^{\prime \prime} .008 \text { rirra. }
$$

Irremo, in generale,

$$
\Phi=\frac{g}{4 . \tau^{-}} \frac{1}{l_{r}} T^{2} \gamma^{\prime},
$$

e poiché, col tentere ali $i$ a zero, $T$ lemele all’ufinito, per angugli ali inclinazione del pendolo sulla verticale tendenti a zero, si avrelshe. $\Phi \rightarrow \infty$.

Lal [1] e la [2] valgono pero soltanto in prima approsimazionr. In esse infalli si preseime dallazione della rigidila del filo. Vealremo che. a molivo della rigrielisa. il periorlo del penclolo la un limile fonito al temelere di $i$ allo zero.

2. - Riferiamoci alla fig. 2. rappresentante un pendolo a so-pensione bifilare, lipo Zälner. Siano $f_{1}, f_{2:}$ lo tensioni relative ai fili $P C, Q B$.

Dovra essere $\left({ }^{1}\right.$ )

$$
\begin{aligned}
f_{1} \cos \hat{\vartheta}_{1}=f_{2} \cos \hat{u}_{2}, \quad f_{1} \sin \hat{\vartheta}_{1} & =J_{2} \sin \mathrm{f}_{2}+m g \\
& =2 m g .
\end{aligned}
$$

Queste relazioni valgrono naluralmente nell’ipolesi che i punli $P$. $Q$. si trovino sulla slessa verlicale. In pallica, lale ipolesi i generalmente sochlisfalla con luona approssimazione. 
Poiche $m g=f ., \sin \gamma_{2}$, consegue

$$
2 \text { lang } \eta_{2}=\operatorname{lang} \vartheta_{1} \text {. }
$$

Si hal senuple

$$
f_{1}>f_{2}
$$

Se $C$ e il centro Iella sharretha $A B=l$, si ha

$$
O C=O P \operatorname{cotg} i{ }_{1}, O B=O Q \operatorname{colg} i_{2} ;
$$

e per $O P=O Q$,

$$
O C \operatorname{langr}_{1} \eta_{1}=O B \text { tang } \gamma_{a},
$$

per eni. per la [4],

$$
O C^{\prime}=\frac{1}{2} O B
$$

Percio il punto O dista da 4 di $\frac{2}{3} l$.

Sulposta la massa $m$ concentrata in $A$, il suo momento d'inerzia rispetto ad $O$ ala cui dista di $\frac{2}{3} l$ ì

$$
I=\frac{4}{9} m l^{*}
$$

Proponiamoei oral di determinare l'analogra della [3], seguendo l'indirizzo di Ishimoto (־)

Supponiamo i fili di sospensione sufficientemente lunghi, affinché essi non diano rhe rlue roppie parallele di corsione: se la rigrilita del filo non i notevole, la forza restitutiva principale, una volta che il pendolo sia spostato dalla stal posizione di equilibrio, $\therefore$ Ia forza di gra-

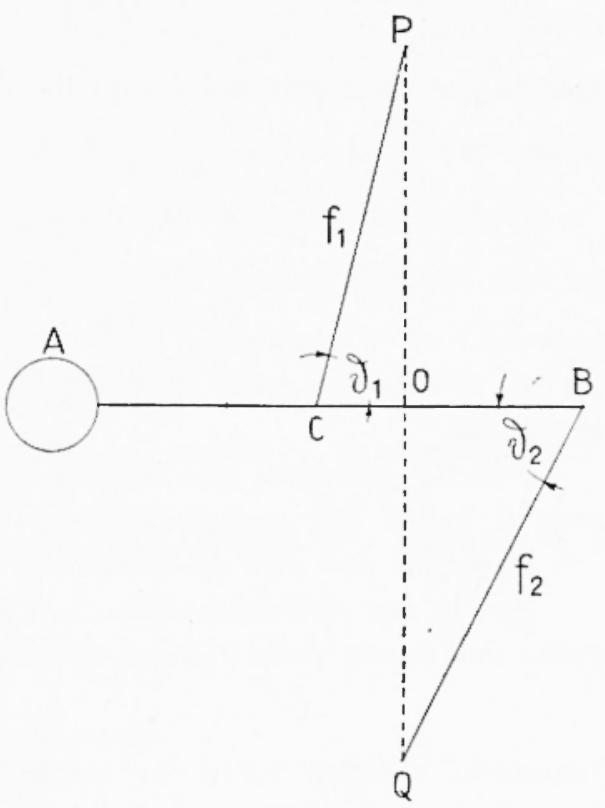

Tig. 2 vili. Vella prima ipotesi, le coppie di torsione del pendolo, per unita d'angolo, salranno

$$
\frac{\pi r^{4} !}{\lambda} .
$$


dove $r$ il raggrio del filo, $u$ la sta rigidita, $\lambda$ la lunghezza media dei lue fili.

Per piecoli spostamenti $i$ dalla verticale, per cui l'azione della gravita diviene $g$ sin $i \supseteq g . i$. l'equazione del movimento diviene

$$
I \frac{d^{2} \omega}{d t^{2}}+\left(\frac{2}{3} m g g i+\frac{\pi r^{t} !}{\lambda}\right) !=0 .
$$

ll periodo del sistema diviene allora

$$
T=2 \pi / \frac{\frac{4}{9} m i}{\frac{2}{3} m g l i+\frac{\pi r^{\prime \prime}}{h}}
$$

Per $\lambda \rightarrow \cdots$, e quindi per coppie di torsione tendenti a zero, la [5] diviene

$$
T = 2 \pi \longdiv { \frac { 2 } { 3 } } \frac { 1 } { g i } .
$$

Questo periodo corrisponde a quello di un pendolo orizzontale, arente il suo punto di rotazione in $O \quad\left(l_{\mathrm{r}}=\frac{2}{3} /\right)$, conformemente alla $[1]$. D'altra parte, per $i=0$, dalla $[5]$ si la

$$
T_{i=0}=\frac{4}{\ddot{\mathrm{j}}} \pi \frac{l}{r^{2}} \mathrm{l} / \frac{\overline{m \lambda}}{\pi ! \iota} .
$$

La [6] ci dice che esiste un limite al periodo e, di conseguenza, alla sensibiliti.

Note le grandezze che in essa figurano, la [6] consente di ottenere il valore del periodo limite.

Passiamo ora alla sensibiliti del pendolo.

Per la sua determinazione, la [2] ra molificata, cosi da tener conto dell'azione della riggidita del filo. Potremo pertanto serivere

$$
\text { 中 }=\frac{y^{\prime}}{i+u} \text {. }
$$

Sostiutuendo $i+a$ a $i$ in [5], si hal

$$
\begin{aligned}
\frac{1}{T^{\sim}} & =\frac{1}{4 \pi^{*}} \frac{\frac{2}{3}-m g l(i+u)+\pi r^{1} \| \lambda}{4 m l^{2} 9} \\
& =K(i+u) .
\end{aligned}
$$


Si osservi che per $\quad \iota=0$ (rigidita nulla) la $\left[5^{\prime}\right]$, che corrisponde appunto al caso di rigidità nulla, dà - per la [7] -

$$
\frac{1}{T^{2}}=\frac{1}{4 \pi^{2}} \frac{3 g}{2 l} \vdots
$$

per cui

$$
K=\frac{3 \varepsilon_{-}}{8 \pi-1}
$$

Dalla [6] invece - per $i=0-\dot{e}$

$$
\frac{1}{T^{-}}=\frac{1}{4 \pi^{2}} \frac{9 \pi r^{1} \mu}{4 m l^{2} \lambda}=\kappa . \mu
$$

cioe

$$
\alpha=\frac{1}{K} \frac{1}{4 \pi^{2}} \frac{9 \pi r^{4} \mu}{4 m l^{2} i}=\frac{3}{2} \frac{\pi r^{4} u}{m g l \lambda} .
$$

La $\left[2^{\prime}\right]$ diviene pertanto - per la $[7]-$

$$
\Phi=K \psi T^{2}=\frac{3}{o} \frac{g}{\pi^{2} i} \psi T^{2} .
$$

Osicrviamo che la [8] é formalmente identica alla [3], nel caso $l_{\mathrm{r}}=\frac{2}{3} l$. Nella [8] però $T$ non tende all'so per $i \rightarrow 0$. Comunque, praticamente, l'una equivale all'altra.

Assegnati $l$ e $y^{\prime}$, la sensibilita del pendolo è determinata unicamente in funzione del periodo $T$.

Se $\delta$ rappresenta la deviazione dell'imagine alla distanza di un metro, arremo

$$
\delta=75-\frac{g}{\pi^{2} l} y \cdot T
$$

3. - Mishio Ishimoto fece costruire il suo pendolo orizzontale, per l'osservazione delle variazioni dell'inclinazione della superficie terrestre, completamente in quarzo, allo scopo di evitare l'effetto delle variazioni di temperatura. L'estrema soltigliezza dei fili (aventi il diametro di 14 micron), rende però questi apparecchi molto fragili e richicde eccessive cautele per l'uso.

Ho ritenuto utile far costruire un pendolo orizzontale che, pur 
rendendo assai piecolo (generalmente trascurabile) l'effetlo della temferatura, consentisse minori preoceupazioni di manegrgio cal una maggrior sicurezza di fumzionamento.

I fili di sospensione sono di costantana, opportunamente rivestiti di sela, con un diametro di 75 micron. Il carico di rollura della co-

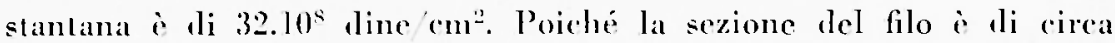
1418 $\iota^{z}$, il carico di rotlura del filo prescelto i pertanto di cirea $1+1370$ line.

D'altra parte, il valore di $l^{1} \dot{c}$ all'incirca $2 m g$ p per $m=\underline{r}$ 14.425 , arremo quindi un fattore di sicurezza allordine di 10.

Eceo le caratleristiche medic ageli apparecehi, che gria fumzionano in parecehie parti d'Italia:

$$
\begin{aligned}
& 11=6.1 \times 10^{11} \text {, line con- (modulo di rigidita della costantana) } \\
& i=\operatorname{lin} 9 \\
& m=\underline{\mathrm{r}} \mathrm{r} \cdot 16, \mathrm{~s} \\
& l=\mathrm{cm} \text { ? } \\
& 2 r=75 \text { micron. }
\end{aligned}
$$

Per $i \rightarrow 0$, il periorlo alel pendolo tenule quindi a 575, come si ricava lalla [6].

Questo tipo di pendolo orizzontale ha gria dato huona prova.

Mi limito qui a riportare un tratto di regristrazione, otlenuta in un concio della dig̣a di sluarramento di un hacino idroclettrico ifigr. 3 .

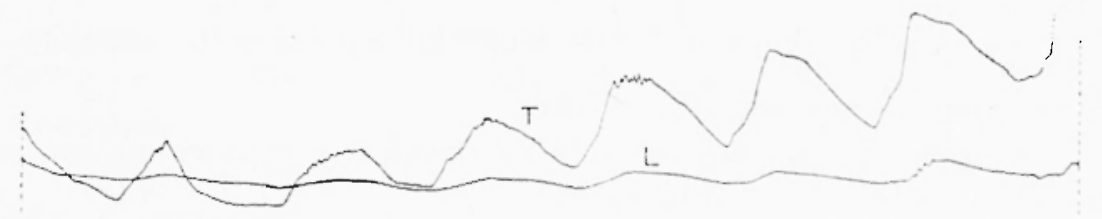

$24 \cdot V \mid \cdot 50$

1. 11150

Fig. 3 - Esempio di registrazione clinografiea. T, spinta di un bacino idroctettrieo verso l'esterno durante l'inwaso; L, componente normale triduzione circa $1 / 3$.

Da essa appare, oltre all'azione lenta dell'invaso in allo fehe si manifesla sotlo forma di spinta verso valle anche londa diuma, dovuta all'azione del Sole sulla parete esposta ai suoi raggi.

Lo strumento è stato costruito presso le ofïcine dell'slituto Naz. di Geofisica, sotlo la guida delladeleto di laboratorio di prima clase geon. Ugro Vacanti, che ha dato realizzazione tecnica al mio progetlo. 
Guesto lipo di apparechio verat sempre piì diffuso fra gli Osservatori della rete geofisica italiana, allestita dall'J.N.G., allo scopo di segruire i lenti movimenti della crosta terrestre.

Roma - Istituto Nazionale di Geofisica - Otıbre 1950.

\section{RI.1SSL ITO}

Si richiamano alcune considerazioni analitiche sull'uso del pendolo orizzontale come clinometro. Si descrivono le caralleristiche fondamentali di un clinometro bifilare, di piccole dimensioni, che ha gia tatolo ottime proce di funzionamento.

\section{BIIBIIOGRAFIA}

(1) Bocasse: 11.: Séismes el sismozraphes - Paris (1927), pag. 137.

(-) Istlunoto M.: Obseriation de linclinaison de la surface terrestre - The Earthquake Researh Institute, Tokso Imperial Lniversity, II (1927). 